\title{
17th RCRA international workshop on "Experimental evaluation of algorithms for solving problems with combinatorial explosion"
}

\author{
Marco Gavanelli • Toni Mancini
}

Published online: 13 January 2012

(C) Springer Science+Business Media B.V. 2012

RCRA (Rappresentazione della Conoscenza e Ragionamento Automatico, rcra. aixia.it) is the interest group on knowledge representation and automated reasoning of the Italian Association for Artificial Intelligence (AI*IA). The annual workshop of the RCRA group is devoted to try to merge the different viewpoints in different communities that try to solve similar problems: those with a combinatorially wide search space. Although the different communities have their own conferences, it is rare to find an event that attracts high quality papers from so different areas as Boolean satisfiability, Answer Set Programming, Scheduling, Planning, using a variety of technology such as Constraint Programming, Lagrangian Relaxation, Local Search, Ant Colony Optimization, and Neural Networks.

The 2010 edition of the RCRA workshop was held in Bologna, in association with $\mathrm{CP}-\mathrm{AI}-\mathrm{OR}$, the international conference on Integration of Artificial Intelligence and Operations Research techniques in Constraint Programming. At the workshop, 17 papers were presented, and the authors had the possibility to submit an extended version of their paper for possible publication in this special issue. After two rounds of reviews, the following nine papers were selected. Marques-Silva et al. [6] study MultiObjective Combinatorial Optimization problems on the Boolean domain with a lexicographic optimization criterion. Cakmak et al. [3] address the problem of finding the best weighted solutions in Answer Set Programming. Oddi et al. [8] present a heuristic algorithm for solving a job-shop scheduling problem with sequencedependent setup times and $\min / \max$ separation constraints among the activities. Gerevini et al. [4] propose an approach to planning with derived predicates where the search space consists of "Rule-Action Graphs". Baioletti et al. [2] introduce

\footnotetext{
M. Gavanelli $(\bowtie)$

Dipartimento di Ingegneria, Università di Ferrara, Via Saragat, 1, 44122 Ferrara, Italy

e-mail: marco.gavanelli@unife.it

T. Mancini

Dipartimento di Informatica, Sapienza Università di Roma, Via Salaria, 113,

00198 Roma, Italy

e-mail: tmancini@di.uniroma1.it
} 
the planner ACOPlan, based on the ant colony optimization framework. Mileo et al. [7] address the problem of indoor position estimation, a central task in homebased assisted living environments, and investigate how state-of-the-art localization and tracking methods can be combined with answer set programming. Pulina and Tacchella [9] present a tool for checking safety of Artificial Neural Networks. Alberti et al. [1] study the problem of reducing the number of observations in applications that require to detect the occurrence of an event in a system. Guimarans at al. [5] present a hybrid approach to solve the Capacitated Vehicle Routing Problem combining a Probabilistic Algorithm with Constraint Programming and Lagrangian Relaxation.

The scientific quality of a scientific publication is mostly due to the quality of the reviews, so we would like to thank all the Program Committee members and the reviewers, who gave constructive suggestions to improve the workshop papers and selected the best ones for this special issue: Stefania Costantini, Luca Di Gaspero, Nicola Di Mauro, Esra Erdem, Ozan Erdem, Halit Erdogan, Stefano Ferilli, Marjin Heule, Jörg Hoffmann, Angel Juan, Nuno P. Lopes, Inês Lynce, Joao Marques-Silva, Alfredo Milani, Alessandra Mileo, Angelo Oddi, Justin Pearson, Valentina Poggioni, Luca Pulina, Kristian Reale, Francesco Ricca, Fabrizio Riguzzi, Carsten Sinz, Marco Sirianni, Terrance Swift, Paolo Torroni, and Pierfrancesco Veltri.

\section{References}

1. Alberti, M., Dell'Acqua, P., Pereira, L.M.: Observation strategies for event detection with incidence on runtime verification: theory, algorithms, experimentation. Ann. Math. Artif. Intell. (2011). doi:10.1007/s10472-011-9259-5

2. Baioletti, M., Milani, A., Poggioni, V., Rossi, F.: Experimental evaluation of pheromone models in ACOPlan. Ann. Math. Artif. Intell. (2011). doi:10.1007/s10472-011-9265-7

3. Cakmak, D., Erdem, E., Erdogan, H.: Computing weighted solutions in ASP: representationbased method vs. search-based method. Ann. Math. Artif. Intell. (2011). doi:10.1007/s10472011-9242-1

4. Gerevini, A., Saetti, A., Serina, I.: Planning in domains with derived predicates through rule-action graphs. Ann. Math. Artif. Intell. (2011). doi:10.1007/s10472-011-9240-3

5. Guimarans, D., Herrero, R., Riera, D., Juan, A.A., Ramos, J.J.: Combining probabilistic algorithms, constraint programming, and lagrangian relaxation to solve the vehicle routing problem. Ann. Math. Artif. Intell. (2011). doi:10.1007/s10472-011-9261-y

6. Marques-Silva, J., Argelich, J., Graça, A., Lynce, I.: Boolean lexicographic optimization: algorithms \& applications. Ann. Math. Artif. Intell. (2011). doi:10.1007/s10472-011-9233-2

7. Mileo, A., Schaub, T., Merico, D., Bisiani, R.: Knowledge-based multi-criteria optimization to support indoor positioning. Ann. Math. Artif. Intell. (2011). doi:10.1007/s10472-011-9241-2

8. Oddi, A., Rasconi, R., Cesta, A., Smith, S.F.: Solving job shop scheduling with setup times through constraint-based iterative sampling: an experimental analysis. Ann. Math. Artif. Intell. (2011). doi:10.1007/s10472-011-9264-8

9. Pulina, L., Tacchella, A.: NeVer: a tool for artificial neural networks verification. Ann. Math. Artif. Intell. (2011). doi:10.1007/s10472-011-9243-0 